\title{
LA DOMINATION \\ DANS LES CONTES DE JACQUES FERRON \\ Martine Jacquot
}

(Acadia University)

Jean Marcel, dans Ferron malgré lui, écrit que le conte est «une entreprise de mise en échec du monde par l'imagination créatrice de façon à faciliter le mode d'insertion de la conscience dans un réel devenu inavouable.» ${ }^{1}$ Sous une apparence fantastique, comique ou tragi-comique, Ferron essaie de faire prendre conscience au lecteur de différents phénomènes psychologiques ou sociaux. Il ne dit pas nécessairement, il illustre en mettant en scène des personnages typés, parfois caricaturaux, ou encore des animaux personnifiés, comme c'est d'ailleurs le cas dans le conte traditionnel. En s'éloignant du réel, le conteur a plus de liberté de mouvement, et le lecteur peut alors observer un phénomène à travers le miroir déformant du conte, voir à travers la loupe de l'écriture. La fantaisie du conte, en déguisant et en maquillant le réel, ne fait que mieux en mettre en valeur certains aspects, certaines facettes. La dénonciation est ainsi facilitée.

Un des thèmes dominants dans les Contes de Ferron est la mise à jour de la domination, que ce soit d'un individu sur d'autres, d'un certain environnement ou d'une certaine société sur l'homme, dans une optique politique, sociale ou religieuse, et nous voyons selon le conte comment réagit le personnage à cette domination.

Le thème de la domination est déjà présent de façon implicite dans le type d'écriture de Ferron. En effet, il a choisi d'écrire en français classique, accusant ainsi la réalité d'une langue menacée d'être dominée par le joual, langue de la ville de Montréal, qui veut envahir la province de Québec. Jacques Ferron déclare lui-même à ce propos que «le joual ne s'écrit pas. S'il a une dignité, cette dignité sera de servir le jargon à une conspiration.» ${ }^{2} \mathrm{Ce}$ thème apparaît plus explicitement dans Retour au Kentucky dans lequel le couple américano-belge cherche un endroit,bilingue où s'installer. L'auteur

LittéRéalité, Vol. II, No. 1, Printemps/Spring 1990 
sous-entend que si, à Lowell, il est trop tard, l'anglais ayant dominé le français, il reste peu de chance pour que le français survive longtemps à Montréal. «La géographie humaine évolue vite en Amérique. Nos livres dataient déjà: Lowell, too late! Nous sommes arrivés à temps à Montréal.» ${ }^{3}$ Mais bientôt, le mari anglophone indique à sa femme francophone que ses enfants seront anglophones. «Ce sont eux qui vous apprendront l'anglais, ma chère! ${ }^{4}$

D'emblée, le lecteur est averti que la condition humaine est moins propice à résister à la domination en ville, où le rythme est aliénant. Le médecin et le curé règlent le cas de la vieille dame en vingt minutes dans $L a$ Dame de Ferme-Neuve. Les notables considèrent comme fous ceux qui réussissent à résister à l'assimilation et aux règles de la société comme on le voit dans Le Retour à Val-d'Or. Par contre, en Gaspésie, le paysagiste dans le conte du même nom réussit à se faire accepter, ou les cochons réussissent à faire comprendre au médecin un peu trop imbu de son rôle qu'ils sont plus humains que lui dans Une Fâcheuse compagnie, pour ne citer que quelques exemples. Il semble donc clair qu'en campagne, la vie est plus humaine, et la survie de l'individu plus réaliste. L'élément comique apporté par les cochons ajoute au ridicule de l'attitude des dominateurs.

L'échec de la domination en Gaspésie est renforcé par la présence de la mer - fenêtre sur l'extérieur, force vitale et protectrice, ouverture de l'esprit s'opposant à l'atmosphère étouffante des villes - présence qui est parfois symbolisée par le goéland, comme dans La Dame de Ferme-Neuve. Le curé et le médecin se sont laissés dominer par la société moderne, mais l'oiseau, lui, est resté libre.

Cette opposition ville-campagne est particulièrement bien illustrée par les personnages du cartographe dans Les Provinces et de l'artiste Jérémie dans Le Paysagiste. Ils se trouvent dans une situation quelque peu homologue, l'un essayant de représenter sa vision du pays sous forme de carte, l'autre de tableau. Mais le cartographe se heurte en ville aux dominations politique et religieuse: le soleil porte une perruque anglaise, la lune une cornette de nonne et les étoiles dans le ciel symbolisent la domination américaine. $\mathrm{Ce}$ conte allégorique démontre que seul contre tous, en ville, l'homme ne peut 
pas lutter. Par contre, Jérémie a moins de peine à s'imposer aux autres car il vit en harmonie avec le paysage, et, par conséquent, il a plus de force. Le rôle de l'artiste est social, il a besoin des autres pour créer et pour exister, et la résistance du milieu ne fait que renforcer sa conviction en son art. Peindre, comme écrire, empêche la réalité de mourir, aide à résister à la domination du temps. Même s'il meurt seul «dans les ruines de son oeuvre,» ${ }^{5}$ l'artiste a réussi à se faire accepter. «Sa grande réussite avait été de se faire accepter par les siens.» ${ }^{6}$ Comme le vagabond dans Martine, qui laisse un certain éclairage sur son passage, «plus de cohérence, ${ }^{7}$ le peintre Jérémie, dont le nom indique particulièrement le rôle de prophète qui montre le chemin de la libération, laisse une image plus claire «dont il laissait provision pour toujours. ${ }^{8}$ L'art rend la réalité plus acceptable, aide l'individu à survivre dans un monde aliéné et dominé. La reconnaissance accordée à l'artiste importe peu, mais son oeuvre et l'impact qu'elle laisse, eux, comptent.

Il est clairement démontré que la sauvegarde du pays est vitale pour la survie de l'équilibre mental et social. Le pays non corrumpu, comme nous l'avons déjà indiqué, est dans cette oeuvre souvent représenté par la Gaspésie, où l'auteur a vécu et dont il garde un souvenir heureux. Cette région apparaît dans Les Cargos noirs de la guerre comme «ces paroisses sans sauvagerie dont le paysage était humanisé depuis longtemps,» ${ }^{9} \mathrm{~s}^{\prime}$ opposant au côté déshumanisé des villes. C'est vers cet espace plus humain que, dans Mélie et le boeuf, Mélie, bernée par son mari et le curé, ramène Maître Leboeuf, un poète suffocant au sein de la ville où l'éducation lui avait fait perdre son identité. Mais l'attachement au pays ne suffit pas. Si le paysan dans Le Vieux payen laisse derrière lui une terre qu'il a travaillée avec autant d'amour que le peintre a mis de lui-même dans son tableau, «humanisant soixante arpents de sauvagerie, ${ }^{10}$ il l'a fait au détriment des siens, qu'il n'a pas aimés, et son travail égoïste disparaît avec lui, contrairement à l'art altruiste qui survit.

L'amour du prochain est un élément essentiel de survie, pas nécessairement dans un sens religieux, mais tout simplement humain. Nous avons vu que le curé et le médecin se sont laissés embarquer dans le tourbillon de la ville dans La Dame de Ferme-Neuve. Il en est de même dans Martine pour ses parents et son ami Jeannot. Son père cède à l'alcool, sa mère 
étouffe et son ami se réfugie dans l'amour du luxe. En se prostituant, elle joue un double rôle: elle symbolise le pays et ses habitants qui cèdent à la société de consommation, qui se prostituent pour survivre, ou bien elle joue le rôle de Marie-Madeleine qui ouvre ses bras aux pêcheurs en perdition. Dans le premier cas, elle perd son identité; dans la deuxième interprétation, elle a résisté à la domination et elle essaie d'aider les hommes égarés. Il existe une troisième lecture, où Martine prend sa revanche sur la société, qui l'a dominée, en dominant à son tour les hommes qui viennent à elle. Dans chacun des cas, son désir de s'échapper mène à une impasse. Elle ressemble aux hommes qui ne trouvent pas de solution en venant la voir. «Ils viennent à moi pour je ne sais quelle délivrance, comme si j'étais la porte du monde.» ${ }^{11}$ Elle ressemble à Mithridate qui n'a plus de rôle à jouer et qui cherche l'exil dans la «robine», ce qui lui évite même d'avoir à prendre la peine de partir. Dans Les Grands soleils, il l'annonçait déjà: «On a appris à fabriquer l'exil sur place. C'est la découverte du siècle.» ${ }^{12}$ Quand l'abrutissement de la ville ou de la banlieue s'ouvre sur une note d'espoir, elle est accompagnée d'un gros point d'interrogation. L'héroïne du Retour à Val-d'Or refuse la domination de la ville. Elle reste chez elle pour éviter l'état de siège psychologique que même son mari subit. Son départ en taxi signifie qu'elle échappe à l'abrutissement de la vie rurale, mais est-il encore temps, pourra-t-elle se réintégrer ailleurs? Sa fuite vers une vie plus saine s'accompagne du sacrifice de l'équilibre familial, puisqu'elle abandonne enfants et mari derrière elle. De même, l'héroïne de La Tasse de thé tourne la page vers le rêve, mais de quel avenir s'agit-il? N'aura-t-elle pas à faire face à un autre type de domination là où elle va?

Cette question nous amène à l'étude d'un autre type de domination, celle de l'homme sur son prochain. La femme est souvent présentée comme asservie par l'homme, les enfants par leur père. La femme est celle dont le mari obtient des enfants: «Un habitant, bon cultivateur, qui avait su obtenir de sa femme treize enfants; ${ }^{13}$ elle est celle que la Mi-Carème bat. Elle ne semble pas avoir, en général, droit à la parole, à la prise de décision. Dans L'Otarie, la femme se présente comme une victime, un objet possédé par son mari. Elle ne vit pas, elle subit et accepte sa condition d'esclave sans réagir: «J'ai fait l'amour de mon mieux, sans beaucoup de satisfaction ni de déplaisir.» ${ }^{14}$ On sait que le même sort attend l'héroöne du Bouquet de noce 
ou encore de Servitude. Armande, dans ce dernier conte, croyant dominer l'homme qui domine son père, se trouve prise au piège. Si elle parvient à monter dans la société, ce ne sera que pour s'enfoncer davantage dans l'impasse psychologique. Elle sera passive et mènera une vie qu'elle n'aimera pas. Il en est de même pour la fille du Chien gris. Dominée par son père, le maître des chiens noirs, elle se retrouve entre les mains de son commis, le maître du chien gris. L'enjeu n'est pas l'amour, mais la possession. En se disputant Nelly, les deux hommes se disputent le domaine, le rôle dominateur. Contrairement au conte Servitude, dans lequel la domination venait de l'extérieur en la personne de Mr. Pas-d'Pouce, ici elle vient de l'intérieur. On pourrait croire que le commis vient délivrer la fille, mais il ne fait que l'enfoncer dans son rôle de femme dominée. Enceinte, elle est prise au piège. Elle passe des mains d'un maître à un autre. Le conte ne se termine pas sur la note classique du «ils vécurent heureux et eurent beaucoup d'enfants,» mais la pauvre fille, qui est d'ailleurs plus présentée comme une chienne que comme une personne, («Son visage semble plus petit que naguère; ses épaules sont renvoyées en arrière et le ventre...» ${ }^{15}$ ) n'est même pas demandée en mariage. Bezeau, le dominateur, se retrouve dominé. «On se rendait compte qu'il était un pauvre homme de soixante ans et plus. Il mourait peu après.» ${ }^{16}$ Le malfaiteur est puni, certes, mais aux dépens d'une tierce personne.

Le mâle dominateur est présent dans La Corde et la génisse, de façon très humoristique. Le capitaine Bove demande qu'on lui apporte une génisse, désignant ainsi une femme. Le marchand Bezeau, quant à lui, parle de sa génisse comme s'il s'agissait d'une femme. Le premier apparaît comme un taureau, un bovin comme son nom l'indique, et le second comme un chien. Tous deux sont ridiculisés par le tour que leur joue Wellie, et l'impact est d'autant plus fort que la bonne du curé, Marguerite - nom souvent utilisé pour nommer une vache - une femme donc, est celle qui tient en fait les ficelles du dénouement. En dénonçant la domination des notables et grâce à l'appel au fantastique, elle sauvegarde l'équilibre entre les hommes. Une fois de plus, la Gaspésie respire.

Pour l'étude de la domination du père sur son fils, nous examinerons trois contes, Cadieu, La Vache morte du canyon et Le Déluge. Dans chacun de ces 
contes, nous avons affaire à une relation père-fils, mais il y a une différence. Cadieu est l'aîné, la société lui donne des droits que François n'a pas. Il poursuit un cheminement semblable à Mithridate et à Martine en ce sens qu'il refuse de se laisser dominer par qui que ce soit. S'il a dû s'exiler de chez lui et changer son nom, il affirme son identité profonde et son indépendance en brûlant la maison paternelle. Par contre, François Laterrière n'est pas l'aîné et n'a droit à rien, pas même à s'affirmer. Poussé hors de chez lui par les pressions sociales et religieuses, il part à la recherche d'un idéal vain symbolisé par la vache morte, but ultime et dérisoire de son errance. François n'est pas armé psychologiquement ou socialement pour se débarrasser des dominations. Il restera, d'une certaine manière, excommunié à jamais des terres que les règles de la société ne lui ont pas attribuées.

Le Déluge est certainement le conte où le thème de la domination est le plus fort. Ni la femme ni les enfants n'ont eu leur mot à dire face à l'homme qui apparaît comme une bête sauvage, armé d'un bâton, symbole phallique par excellence: «la face broussailleuse et le bâton entre les jambes, sur lequel il se soulevait à l'occasion pour mieux voir les bêtes et autres créatures de passage.» ${ }^{17}$ Insatiable, il cherche à dominer son fils héritier en pensant à séduire sa belle-fille, mais le célibat du jeune homme le pousse vers les villes où les «créatures» abondent. Le dominateur parti, la maison paternelle, dont le rôle était de protéger la famille, telle une arche, n'a plus de raison d'être. Elle s'en va à la dérive, ce qui signifie peut-être que la génération d'opprimeurs disparaît, s'en va vers les villes.

Pour en revenir à l'auteur, conscient du phénomène de domination qui menace l'individu et le pays de toutes parts, il a choisi d'écrire pour pallier à cet état de siège. Il écrit le pays pour lui redonner son identité, il s'écrit pour garder contrôle sur lui-même et sur ce qui l'entoure, pour ne pas se laisser corrompre ou dominer par le tourbillon de la vie. Dans La Dame de FermeNeuve, il note très clairement: «Moi entre deux nuits, qui écris pour me remettre à jour.» ${ }^{18}$

Dans l'oeuvre de Ferron, le dominateur s'appelle souvent Baal, Papa Boss ou Minotaure. Il est le symbole des grands empires économiques qui dominent le monde, le transforment en monde de consommation. Celui qui 
a connu le monde avant sa transformation, c'est le conteur, celui qui offrait sagesse et fantaisie. Avec l'expansion des villes, qui débordent dans les campagnes, le conteur est désormais étranger dans un cadre naguère familier. Son échec entraîne celui des hommes qui n'ont plus de guide. «L'homme seul est pour lui-même une proie. La solitude est signe de notre temps. Il n'y a plus d'échange, il n'y a plus de société. C'est la règle d'un malentendu grotesque, et, dans l'air raréfié le néant frôle le monde.» ${ }^{19}$ Les mots «malentendu» et «frôle» dans la bouche de Ferron-conteur laissent toutefois une note d'espoir. Le malentendu peut se résoudre. Qui dit «frôler» ne veut pas dire «dominer».

\section{边}

\section{NOTES}

${ }^{1}$ Jean Marcel, Jacques Ferron malgré lui (Montréal: Editions du Jour, 1970), p. 54.

2Jacques Ferron, Montréal: Le Devoir (Montréal, 30 octobre 1967), p. 17.

${ }^{3}$ Jacques Ferron, Contes (Montréal: Editions HMH Ltée, 1968), p. 150.

${ }^{4}$ Ibid., p. 150.

${ }^{5} I$ Ibid., p. 61.

${ }^{6} I b i d .$, p. 59.

${ }^{7}$ Ibid., p. 125.

${ }^{8}$ Ibid., p. 61.

${ }^{9} I b i d .$, p. 157.

${ }^{10}$ Ibid., p. 147. 
${ }^{11}$ Ibid., p. 129.

${ }^{12}$ Jacques Ferron, Les Grands soleils (Montréal: Librairie Déom, 19...), p. 28.

${ }^{13}$ Jacques Ferron, Contes (Montréal: Editions HMH Ltée., 1968), p. 111.

${ }^{14}$ Ibid., p. 152.

${ }^{15} \mathrm{Ibid} ., \mathrm{p} .71$.

${ }^{16} \mathrm{Ibid}$. , p. 73.

${ }^{17}$ Ibid., p. 112.

${ }^{18}$ Ibid., p. 179.

${ }^{19}$ Ibid., p. 129.

\section{Q3 \\ BIBLIOGRAPHIE}

Boucher, Jean-Pierre. Les «Contes» de Jacques Ferron. Montréal: Les Editions de l'Aurore, 1974.

Ferron, Jacques. Contes. Version intégrale. Montréal: Editions HMH Ltée., 1968.

Ferron, Jacques. Les Grands soleils. Montréal: Librairie Déom, 19....

Marcel, Jean. Ferron malgré lui. Montréal: Editions du Jour, 1970.

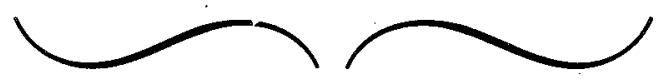

